\title{
Aplicación GPS para administración de servicios
}

\section{GPS application for service management}

MEDINA-VELOZ, Gricelda $\dagger^{*}$, LUNA-ROSAS, Francisco Javier, TAVAREZ-AVENDAÑO, Juan Felipe y DORADO-PUGA, Bryant Arturo

\author{
Universidad Tecnológica del Norte de Aguascalientes, Aguascalientes Ags., México \\ Instituto Tecnológico de Aguascalientes, Aguascalientes Ags, México. \\ ID $1^{\text {er }}$ Autor: Gricelda, Medina-Veloz / ORC ID: 0000-0002-1955-3620, arXiv Author ID: GrisArvix18, CVU CONACYT \\ ID: 228438 \\ ID: 1er Coautor: Francisco Javier,Luna-Rosas / ORC ID: 0000-0001-6821-4046, arXiv Author ID: arXivFco19, CVU \\ CONACYT ID: 87098
}

ID 2do Coautor: Juan Felipe, Tavarez-Avendaño /ORC ID: 0000-0001-8336-1546

ID 3er Coautor: Bryant Arturo, Dorado-Puga

DOI: $10.35429 /$ JCA.2019.9.3.11.17

Recibido Enero 10, 2019; Aceptado Marzo 30, 2019

\section{Resumen}

El continuo desarrollo tecnológico al que estamos expuestos en la actualidad, avanza a una velocidad sin precedentes, cambiando muchos aspectos de nuestra vida cotidiana. Dentro de estos avances se encuentra el uso de sistemas de geolocalización. Con los cuales es posible, conocer la ubicación exacta de elementos estáticos o móviles, la programación de desplazamientos, el cálculo de distancias y tiempos de recorridos, el control de donde se encuentran en cada momento objetos y personas, y hasta llamadas de auxilio SOS. Este tipo de sistemas de software de geolocalización, proporcionan datos e información muy útiles para numerosas funcionalidades y aplicaciones en la sociedad. Con este proyecto, se pretende mejorar la productividad de los empleados de la empresa CFE estación central de Aguascalientes, mediante la implementación de un software GPS que le muestre al empleado la ubicación exacta y el tipo de servicio que va a realizar, mediante la visualización de un mapa que le indique la ruta a seguir para encontrar el punto exacto del servicio a desarrollar, además de registrar los datos del servicio proporcionado para su mejor control y administración.

GPS, Georeferencia, NoniGPSPlot

\begin{abstract}
The continuous technological development to which we are currently exposed, advances an unprecedented speed, changing many aspects of our daily life. Within these advances is the use of geolocation systems. With which it is possible to know the exact location of static or mobile elements, the programming of trips, the calculation of distances and times of routes, the control of where objects and people are all times, and even SOS calls for help. This type of geolocation software systems provide very useful data and information for numerous functions and applications in society. With this project, the aim is to improve the productivity of the employees of the company CFE Aguascalientes central station, by implementing a GPS software that shows the employee the exact location and the type of service that will be carried out, by visualizing the a map that indicates the route to follow to find the exact point of the service to be developed, in addition to register the data of the service provided for its better control and administration.
\end{abstract}

GPS, Geolocation, NoniGPSPlot

Citación: MEDINA-VELOZ, Gricelda, LUNA-ROSAS, Francisco Javier, TAVAREZ-AVENDAÑO, Juan Felipe y DORADO-PUGA, Bryant Arturo. Aplicación GPS para administración de servicios. Revista de Cómputo Aplicado. 2019, 3 9: $11-17$

\footnotetext{
* Correspondencia del Autor (gricelda.medina@utna.edu.mx)

$\dagger$ Investigador contribuyendo como primer Autor.
} 


\section{Introducción}

Un sistema GPS funciona mediante una red de satélites en órbita, con trayectorias sincronizadas para cubrir toda la superficie de la Tierra. Cuando se desea determinar una posición, el receptor localiza automáticamente la señal que indica la identificación y la hora del reloj de su ubicación. Con base en estas señales, el aparato sincroniza el reloj del GPS y calcula el tiempo que tardan en llegar las señales al equipo, de tal modo mide la distancia al satélite mediante el método de trilateración inversa, la cual se basa en determinar la distancia de cada satélite respecto al punto de medición. Conocidas las distancias, se determina fácilmente la posición relativa respecto a los satélites. Conociendo además las coordenadas o posición de cada uno de ellos por la señal que emiten, se obtiene la posición absoluta o coordenada reales del punto de medición. También se consigue una exactitud extrema en el reloj del GPS, similar a la de los relojes atómicos que llevan a bordo cada uno de los satélites.

\section{Sistemas de Software GPS}

En la actualidad, la importancia de los sistemas de software de georeferencia, son tan comunes e importantes, que ya no se consideran como una opción, sino como una necesidad. Existen numerosas aplicaciones open source en el mercado que permiten el aprovechamiento de sus herramientas por una multitud de usuarios, entre las cuales se encuentran: Google Maps Mobile, GPSToday,Waze, Nav4All, NoniGPSPlot entre otras.

Para el caso específico de NoniGPSPlot, que es la herramienta seleccionada para el desarrollo de la aplicación, éste es un intérprete de señales GPS especialmente diseñado para guardar rutas y luego visualizarlas. El programa guarda y dibuja la ruta que se sigue, y una vez el recorrido finalizado, se pueden exportar tus rutas a KML y visualizarlas en el popular visor de mapas, Google Earth.

\section{Funcionamiento general de un sistema GPS}

Un sistema GPS, funciona por medio de 24 satélites (conocidos como NAVSTAR), que constantemente están en órbita alrededor del globo terráqueo.
Estos 24 satélites más 3 de respaldo, rodean la tierra en seis diferentes direcciones. Esto ocurre, para que puedan tener una mejor cobertura del globo, actualmente, cada satélite, da dos vueltas a la tierra por día, lo que ayuda a lograr una mayor precisión al sistema GPS.

Para que un sistema GPS pueda ubicar un punto específico en un mapa, se basa en la medición de distancia entre el receptor y al menos 3 satélites. El receptor mide la distancia al primer satélite y establece una circunferencia imaginaria cuyo radio será esa distancia, permitiendo la ubicación en cualquier punto de la esfera. El receptor toma la distancia que lo separa del satélite 2 y forma nuevamente una circunferencia, donde la ubicación seleccionada estará en la intersección de ambas esferas. Finalmente el tercer satélite crea una nueva esfera que corta en dos puntos la intersección resultante de las otras dos.

El receptor recibe la información de esos 3 satélites y las características de sus órbitas, procesa los datos y obtiene la ubicación exacta con un mínimo margen de error, Imagen 1. Finalmente tras obtener la posición exacta, se introduce al GPS la dirección a donde se desea trasladar y éste que tiene incorporado un ordenador con un mapa busca el mejor camino de para llegar a el.

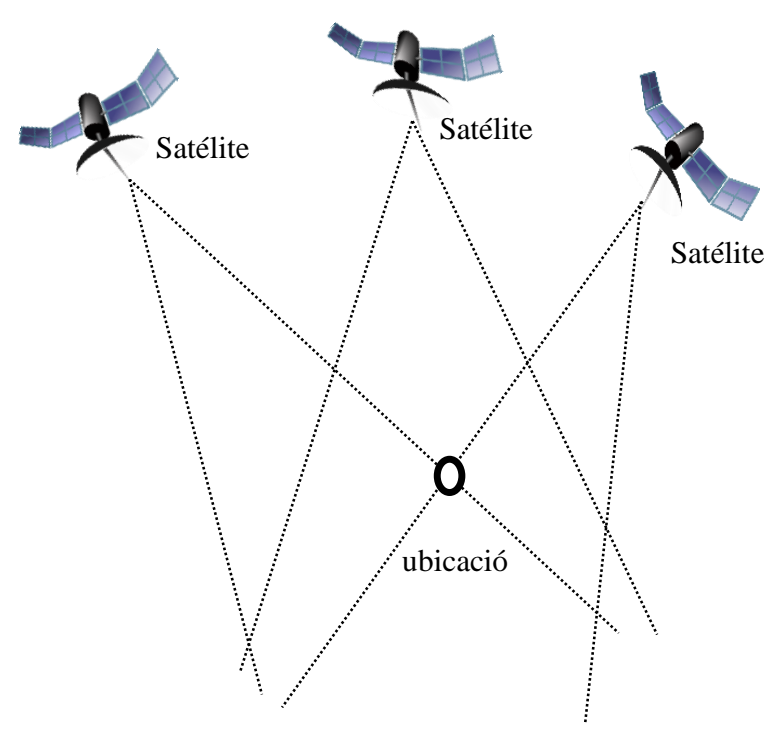

Figura 1 Trilateración 


\section{Problemática y Justificación del proyecto.}

El método de trabajo de los empleados de la CFE estación central, se lleva a cabo mediante una terminal portátil en la cual se guardan las rutas estáticas donde deben realizar los diversos servicios, tales como chequeos, mediciones, revisión de instalaciones o cortes, entre otros. Las terminales cuentan con un sistema operativo Windows Mobile por lo cual se optó por desarrollar una aplicación compatible con este sistema operativo, ya que en esta plataforma se encuentra la herramienta indicada para crear el programa a desarrollar.

El principal problema, es que en la terminal portátil con la que cuentan los trabajadores solo se especifica la dirección a la cual deben ir a realizar el servicio, pero no se tiene alguna referencia visual de su ubicación, y algunas veces resulta complicado al trabajador encontrar el destino al cual debe trasladarse para desarrollar el servicio, provocando con ello retrasos y pérdidas de tiempo con los demás servicios que tiene asignados durante el día disminuyendo en ocasiones considerablemente su productividad.

Otro de los problemas que se encuentran es que, no cuentan con un formato en digital para poder plasmar cada una de las actividades que realizan en los servicios, debido a que todo esto se llena por escrito en una hoja de papel lo que en muchas ocasiones es bastante tedioso y complicado, ya que las hojas se maltratan disminuyendo su visibilidad y en algunas ocasiones se llegan a perder, y con esto se pierde el registro del servicio, el cual es útil para la probar el cumplimiento del mismo además de su inclusión en los reportes y estadísticas, que la empresa debe realizar de forma periódica para realizar sus planes de trabajo a futuro.

Otro de los problemas con los que actualmente se enfrenta la administración de la estación central, es el desconocimiento del uso que se da a los vehículos de la empresa por parte de su personal, ya que al no tener una referencia exacta de la ubicación del servicio, los empleados argumentan las pérdidas de tiempo y los retardos a esta cuestión, ocasionando con ello el gasto excesivo de combustible y poca productividad laboral.
Por lo anterior, lo que se pretende es desarrollar, es una aplicación que indique el punto exacto del servicio sobre una imagen, con lo cual se facilitará la tarea de traslado a cada uno de los trabajadores, así como la creación de formatos digitales que faciliten el llenado de cada uno de los servicios que realizan agilizando con esto sus tareas. Al disponer de un sistema de georeferencias mediante GPS, los trabajadores ya no tendrán dificultad para conocer las rutas diarias del recorrido de los servicios que deberán de realizar, y con el registro en digital se podrán justificar sus actividades diarias y con ello realizar una mejor planeación de los servicios a futuro.

\section{Desarrollo del proyecto}

Primeramente, se realizó una investigación en relación a los tipos de formatos de archivo de las aplicaciones que sorportaba la terminal física, con la cual se va iba a trabajar, en este caso archivos con extensión .CAB, así como la investigación de herramientas previamente desarrolladas y lanzadas al mercado, que cumplieran con los requisitos del cliente. A continuación en la Figura 2 se muestra la terminal física con la cual los empleados de la empresa realizarían la toma de lecturas y el registro de los servicios que tendrían a su cargo.

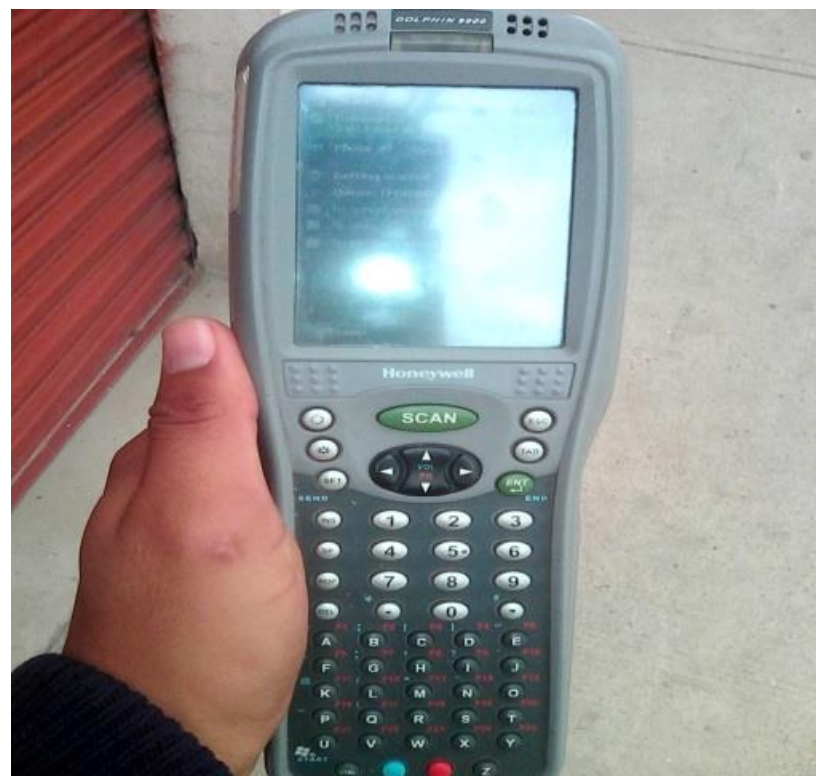

Figura 2 Terminal de lectura

En la siguiente imagen, se puede observar el explorador gráfico de la terminal, por medio del cual se pueden buscar los archivos que contiene en la memoria, para poder instalar las nuevas aplicaciones con las que se planea trabajar. 


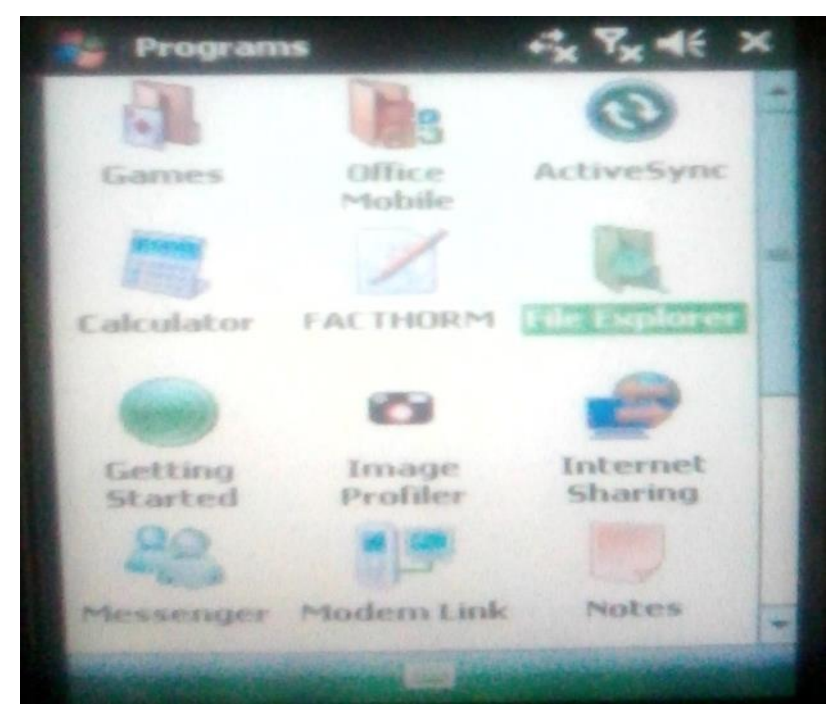

Figura 3 Interfaz de la terminal de lectura

Una vez hecha la investigación sobre el software alternativo de georreferenciación que se podría utilizar, se dio inicio con la elaboración de un prototipo para el desarrollo de la aplicación, con el uso de una herramienta gratuita, que cumplía con los requisitos del cliente. La aplicación que se encontró lleva por nombre Noni GPSPlot. La cual permite cargar mapas con extensión .map y cargar una serie de puntos con georreferencias creando las rutas que se deben de seguir para llegar a un punto específico.

Después de que se instaló el software Noni GPSPlot en las terminales de los empleados, se genera un menú inicial que se puede observan en la Figura 4, que muestra diferentes opciones a elegir tales como, ver la lista de puntos cargados, configurar la aplicación para las georreferencias y mapas, localizar la ubicación actual vía satélite entre otras.

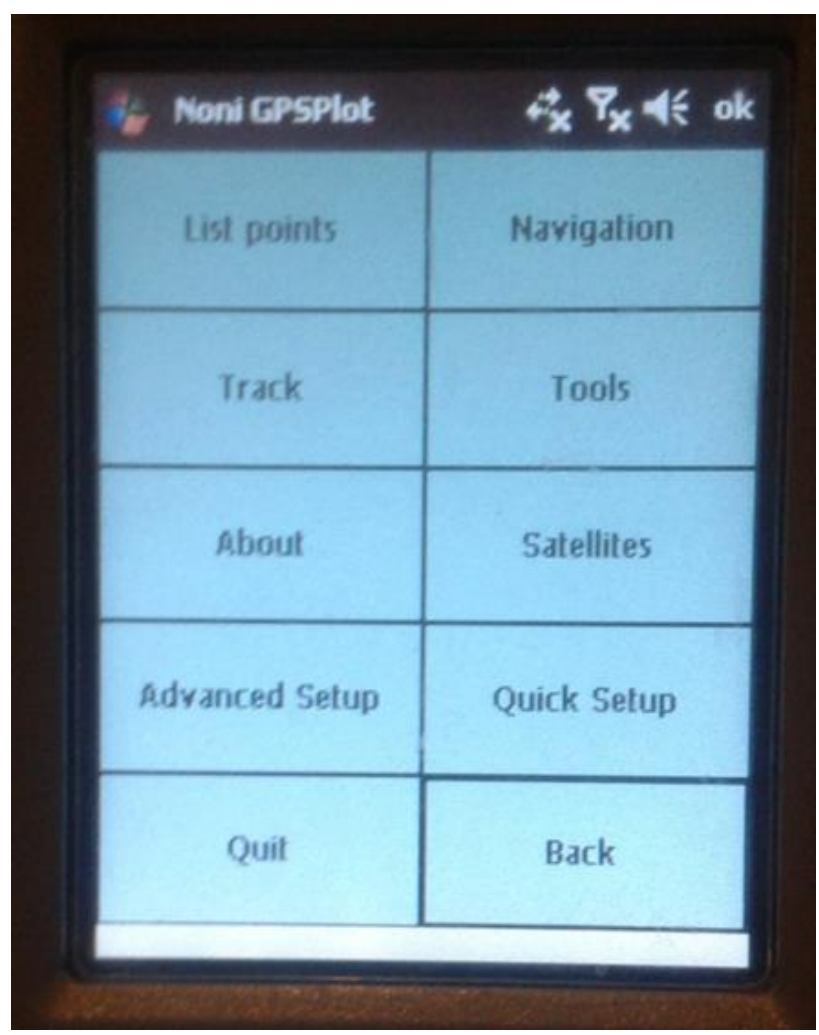

Figura 4 Instalación del Software Noni GPSPlot

Una vez habiendo ingresado a la opción de navegación en el software, se puede observar mediante una Figura satelital (Figura 5), un punto en específico en un mapa previamente seleccionado, el cual indica la ubicación de un servicio que se deberá de llevar a cabo, por los empleados de la empresa.

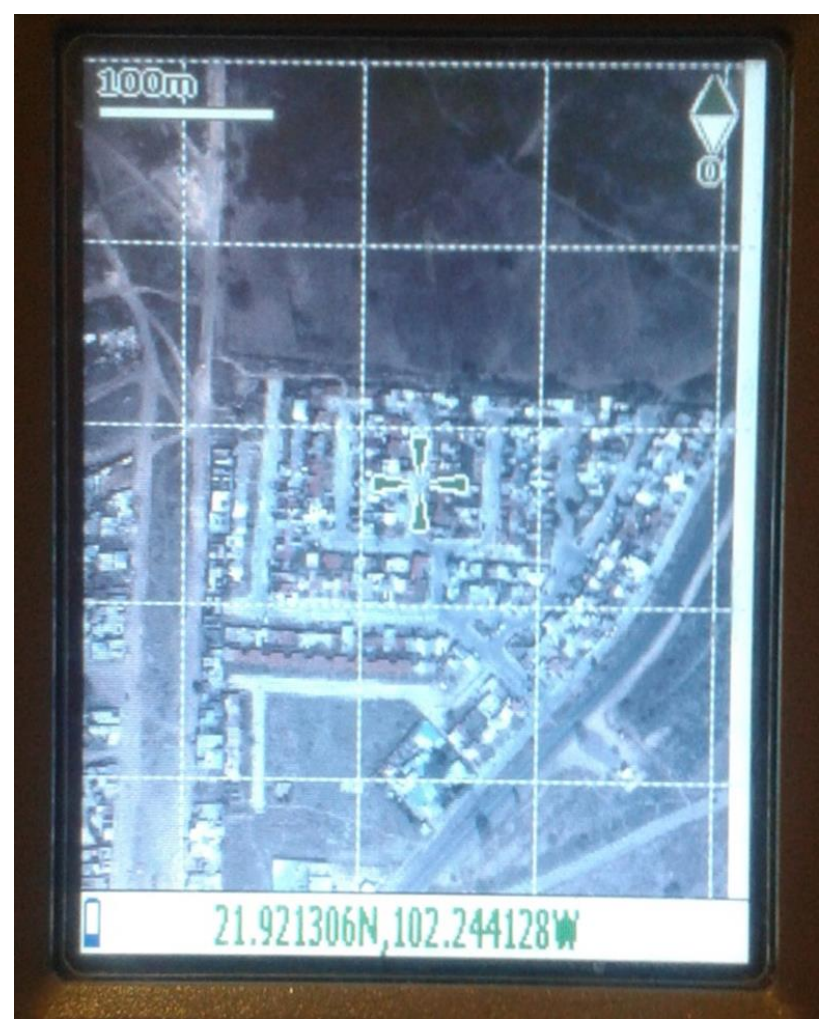

Figura 5 Interfaz de la terminal de lectura, indicación de punto específico de servicio a desarrollar 
Otra de las opciones de la aplicación, es visualizar un conjunto de puntos cargados sobre el mapa, creando una ruta entre ellos, que indica la ruta de los servicios que deberán ser cubiertos en un área determinada por los trabajadores asignados a este cargo. Figura 6

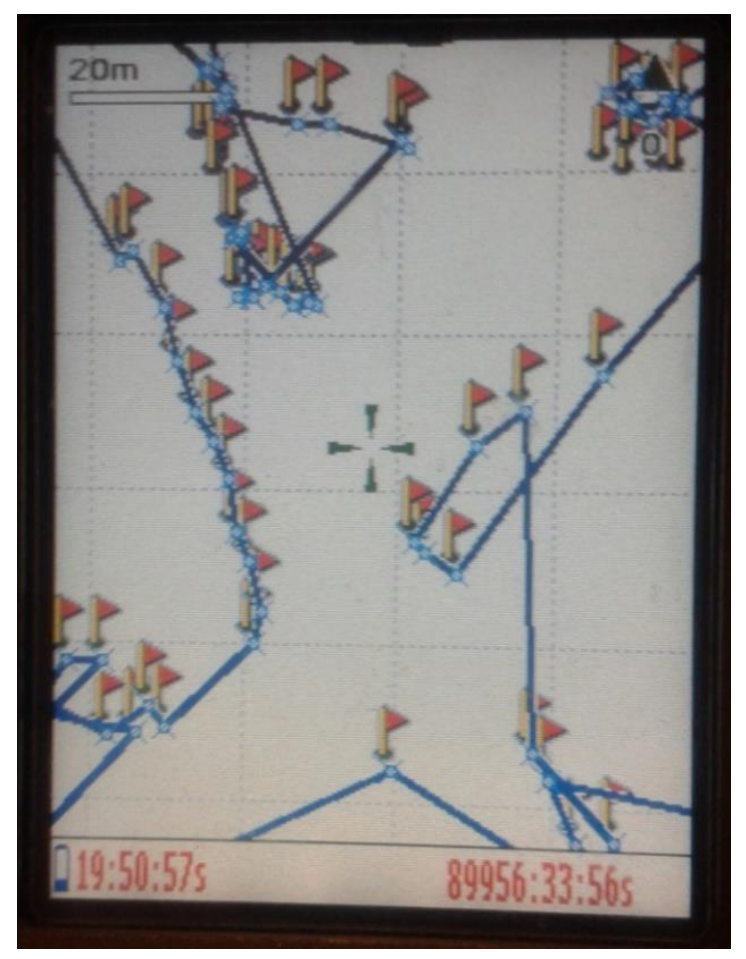

Figura 6 Ruta de servicios

En la siguiente Figura se muestra la lista de archivos que quedan cargados en la terminal, los cuales contienen las georeferencias de cada punto marcado en el mapa que integraron los puntos de servicios en la ruta a realizar, y que serán visibles al momento de cargar el mapa en el software de la terminal. Figura 7

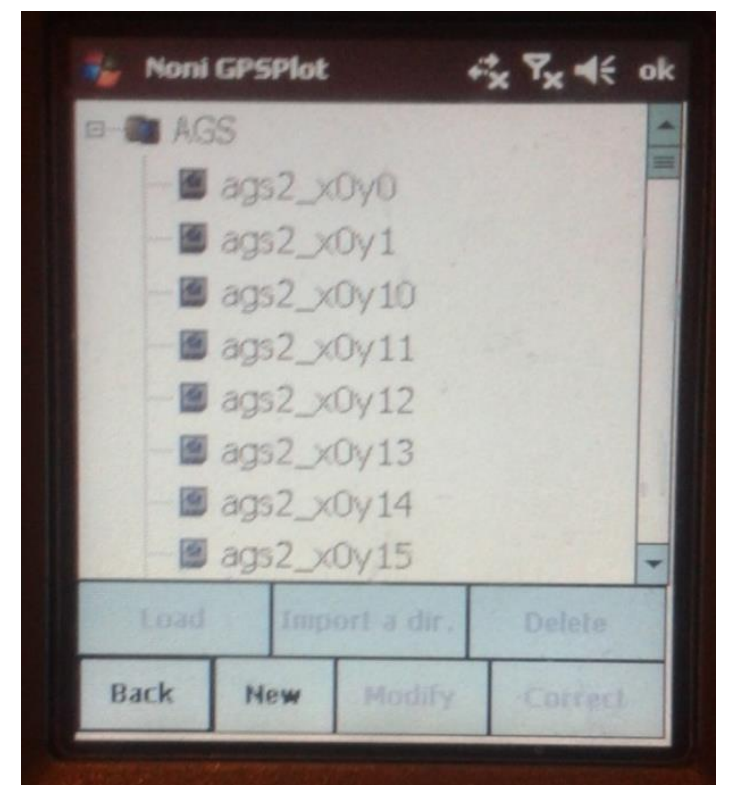

Figura 7 Listados de archivos con georeferencias que integran la ruta de servicios
Para el registro del servicio en el formato digital, se creó el siguiente menú, que se muestra en la Figura 8. Con el cual los usuarios pueden elegir las opciones de: Registro de supervisión, cancelación, anomalías y toma de lectura dependiendo del tipo de servicio que los empleados deseen registrar.

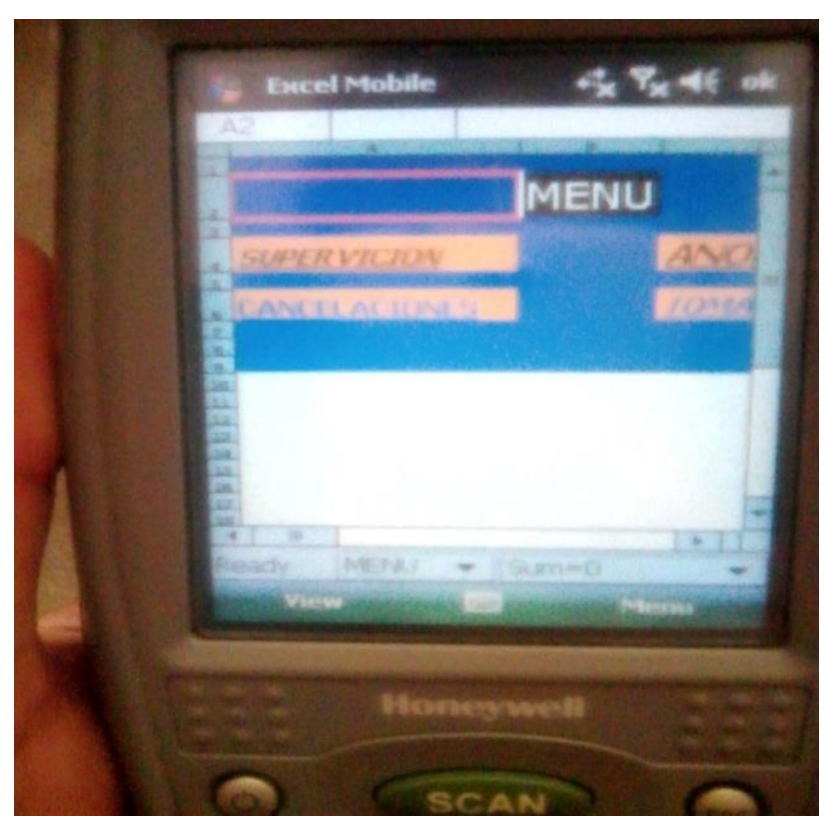

Figura 8 Menú para el registro de servicios

Si el empleado por ejemplo, elige la opción de toma de lectura, aparecerá la siguiente interfaz de captura para su registro en digital, con el cual deberá ingresar los datos del servicio de toma de lectura realizado. Figura 9.

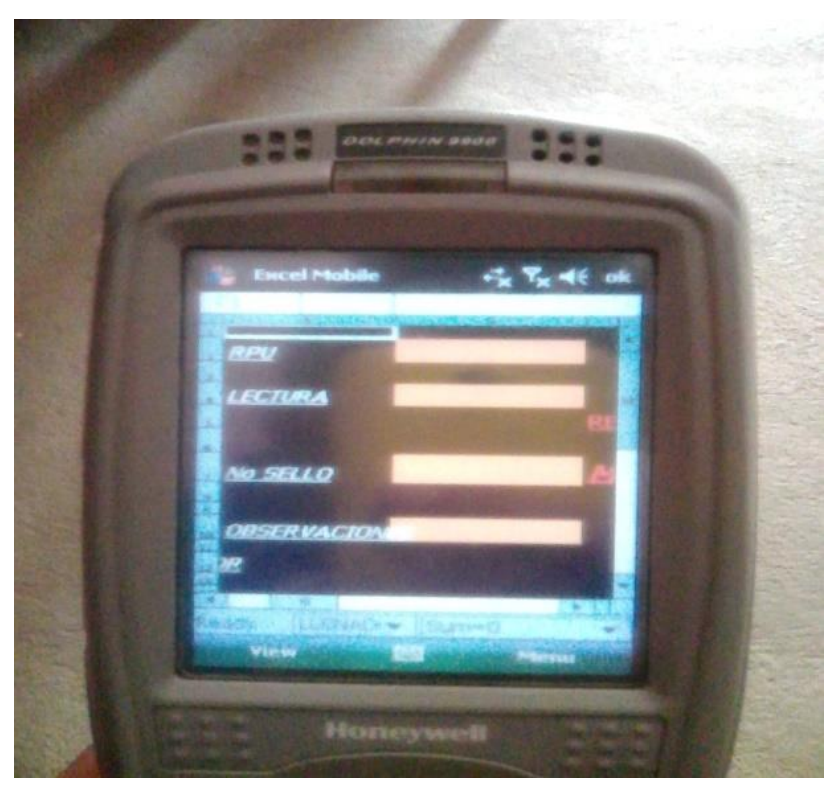

Figura 9 Pantalla para el registro de servicio toma de lectura 
Otra de las opciones que se pueden elegir en la aplicación, es la de generar al término de la jornada laboral, el reporte final de servicios realizados, cuya interfaz se puede observar en la siguiente imagen. Figura 10

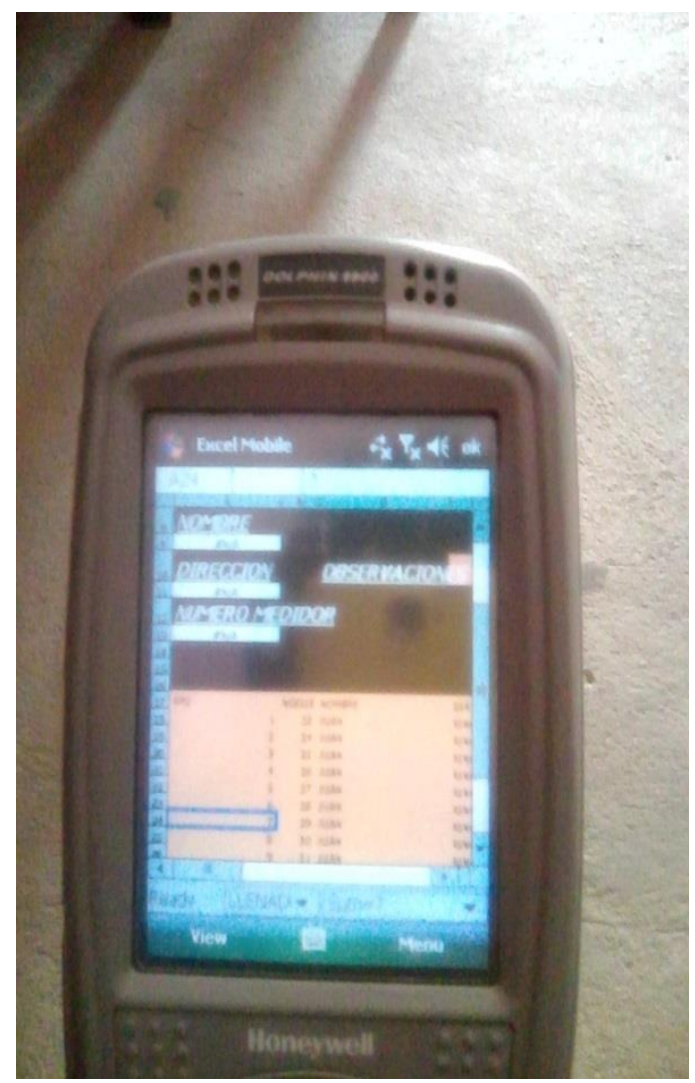

Figura 10 Reporte final de servicios

A continuación se muestra el diagrama de flujo de la aplicación, que representa el proceso de cómo el software desarrollado interactúa con los empleados. Figura 11 En él se puede observar, que el uso de la aplicación inicia con el ingreso de las rutas que el empleado va a cubrir durante su jornada laboral, después de ello, el software verifica y genera las georeferencias de las rutas e inicia con la navegación para luego mostrar la ubicación de cada punto georeferenciado, si el lugar no se localiza, se procede a corregir el error, el cual se puede generar por varias razones, entre las cuales se encuentran, la falta de actualización del software, una mala conexión a Internet, la configuración de los permisos de navegación etc. Una vez corregidos los errores se actualiza la bitácora del sistema y se entrega nuevamente el equipo para reiniciar la navegación. Si la ubicación se muestra en la aplicación se siguen los puntos de la ruta y se registra el servicio.

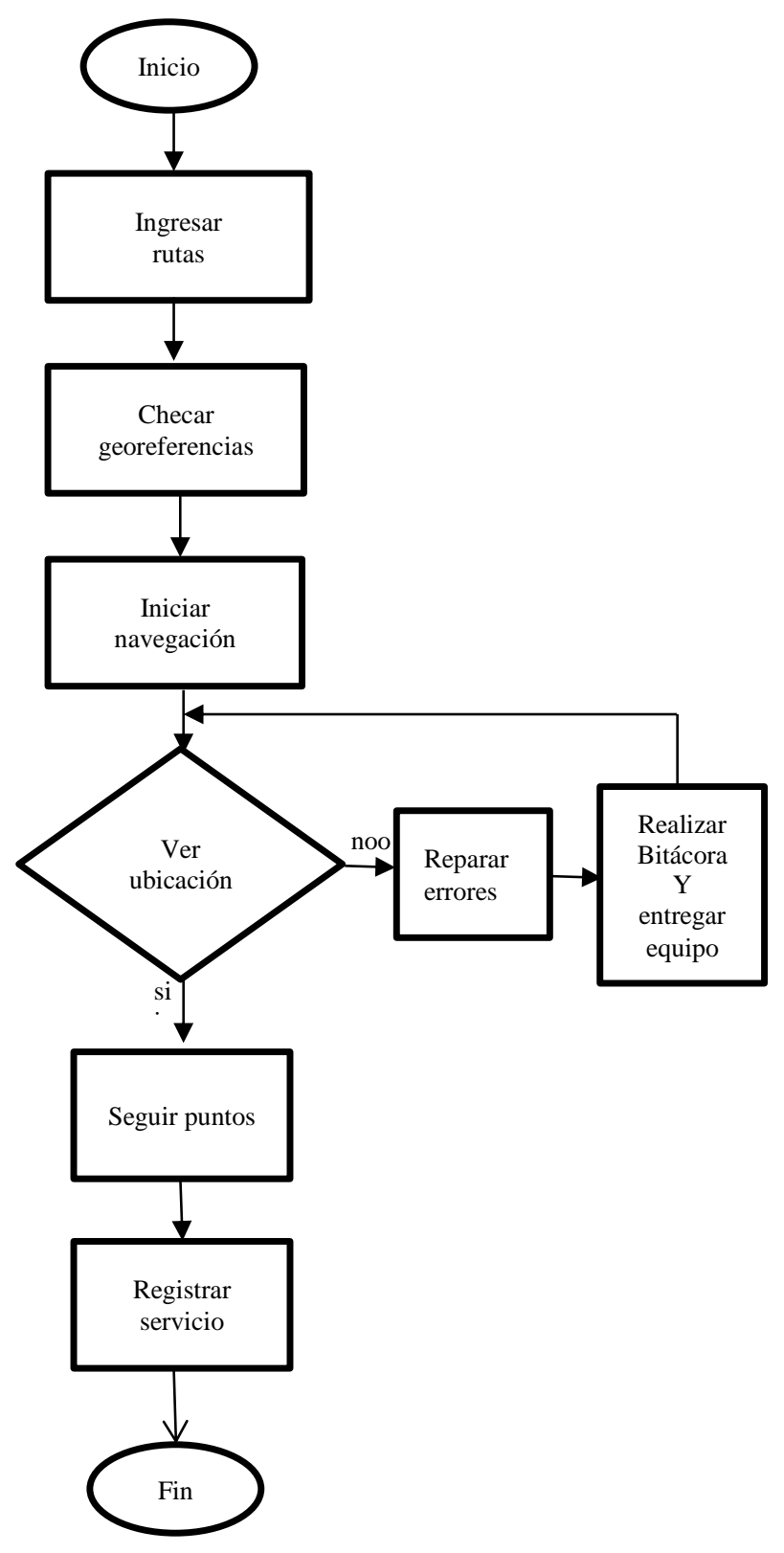

Figura 11 Diagrama de flujo de la interacción del software con los empleados

\section{Resultados y concusiones}

Con el desarrollo del proyecto, se cumplió el objetivo principal que se tuvo planeado al inicio del proyecto, ya que con el desarrollo del sistema GPS y control de servicios, se ha dado a la empresa Comisión Federal de Electricidad estación central. Un software que con el que los trabajadores de línea pueden ubicar rápidamente los medidores que tienen asignados para dar servicios, y de igual manera se pueden generar y guardar los registros de los servicios que se tienen en todas las zonas que ellos tienen a su cargo, llevando acabo un registro ágil y más rápido. 
Cabe señalar que al contar con el sistema que lleva el control y registro de los proyectos se genera un histórico de todos los servicios realizados, ya que estos se quedan guardados para futuras aclaraciones o simplemente para historial y beneficio de los clientes de la misma empresa.

\section{Referencias}

Bocanegra Ureta, R. G. (2012). Desarrollo de una aplicación Web para el monitoreo de vehículos con dispositivos GPS que comercializa una Empresa de Telecomunicaciones.

Choqque, E., \& Americo, C. (2019). Desarrollo de un aplicativo móvil para el monitoreo de trabajadores utilizando la tecnología GPS móvil en la Universidad Peruana Unión Filial-Juliaca.

Contreras, I. C., \& Gómez, G. H. (2018). SISTEMA DE LOCALIZACIÓN EN TIEMPO REAL MEDIANTE UN SERVIDOR WEB Y APLICACIONES MÓVILES. Pistas

Educativas, 39(127).

Fernández Herrera, M. (2019). Geolocalización en el espacio para la gestión de inventario.

Guerrero Machado, F. D. (2019). Sistema de información de rutas y posicionamiento de vehículos oficiales de la Universidad Técnica De Ambato (Bachelor's thesis, Universidad Técnica de Ambato. Facultad de Ingeniería en Sistemas, Electrónica e Industrial. Carrera de Ingeniería Electrónica y Comunicaciones).

Herrera Rosado, R. F. (2011). GPS aplicado a la ubicación de vehículos de transporte terrestre y sus alternativas en su gestión.

Huerta, E., Mangiaterra, A., \& Noguera, G. (2005). GPS. Posicionamiento Satelital.

NoniGPSPlot,

http://aeguerre.free.fr/Public/PocketPC/NoniGP SPlot

Peñafiel, J., \& Zayas, J. (2001). Fundamentos del sistema GPS y aplicaciones en la topografía. Publicaciones del Colegio Oficial de Ingenieros Técnicos en Topografía.
Perez-Mena, A., Fernández-Zepeda, J. A., Rivera-Caicedo, J. P., \& Avila-George, H. (2019). Una aplicación móvil para el monitoreo de cultivos: caso de estudio campaña contra el pulgón amarillo del sorgo. RISTI-Revista Ibérica de Sistemas e Tecnologias de Informação, (31), 118-133.

Quiñonez, Y., Lizarraga, C., Peraza, J., \& Zatarain, O. (2019). Sistema inteligente para el monitoreo automatizado del transporte público en tiempo real. RISTI-Revista Ibérica de Sistemas e Tecnologias de Informação, (31), 94105 .

Rojas, H., \& Alexander, D. (2019). Aplicación de la normativa eléctrica para la individualización del suministro provisional colectivo de venta en bloque en media tensión del caserío Algodonal en el distrito de Paimas provincia de Ayabaca, Piura dentro de la zona de responsabilidad técnica de Electronoroeste SA.

Romero, C., Gonzalo, F., \& Turpo Ticona, F. (2015). Análisis e implementación de un sistema de geolocalización, monitoreo y control de vehículos automotrices basado en protocolos gps/gsm/gprs para la Ciudad de Puno.

Vázquez Seisdedos, C. R., Palacios Meléndez, E. F., Córdova Rivadeneira, L. S., \& Romero Paz, M. (2016). Dispositivo de adquisición y transmisión de la posición de un vehículo mediante GPS y Wi-Fi. Ingeniería Electrónica, Automática y Comunicaciones, 37(2), 15-27.

Vergara Miranda, H. J., Loayza, L., Enrique, J., Bazán Portela, J. A., Flores Gálvez, L. Y., \& Correa Araque, M. M. (2019). Plataforma multilateral para servicios de belleza a domicilio App Salón Express. 